\begin{tabular}{c} 
Volume and Issues Obtainable at Center for Sustainability Research and Consultancy \\
Journal of Accounting and Finance in Emerging Economies \\
ISSN: 2519-0318 ISSN (E) 2518-8488 \\
Volume 6: Issue 1 March 2020 \\
CSRᄃ \\
Journal homepage: www.publishing.globalcsrc.org/jafee \\
\hline
\end{tabular}

\title{
Testing the Validity of Purchasing Power Parity Theory and Dynamics of Exchange Rate Behavior (Pakistan, China, Iran and Turkey)
}

\author{
${ }^{1}$ Rana Shahid Imdad Akash, ${ }^{2}$ Muhammad Mudasar Ghafoor, ${ }^{3}$ Navid Ahmed \\ ${ }^{1}$ Assistant Professor, School of Business Management, NFC-IEFR, Faisalabad, Pakistan: \\ shahid.imdad@yahoo.com \\ ${ }^{2}$ Assistant Professor, University of the Punjab, Jhelum Campus, Jhelum. Pakistan: mudasar@ pugc.edu.pk.com \\ ${ }^{3}$ Lecturer IMS, University of Agriculture, Sub Campus Toba Tek Singh, Pakistan: navid_mcom@yahoo.com
}

\begin{tabular}{l}
\multicolumn{1}{c}{ ARTICLE DETAILS } \\
\hline History \\
Revised format: February 2020 \\
Available Online: March 2020 \\
\\
\hline Keywords \\
Purchasing Power Parity, Law \\
of One Price, Long Run, Short \\
Run
\end{tabular}

JEL Classification:

K12, K19, F20

\begin{abstract}
Purpose: This study is aimed at to observe the purchasing power parity (PPP) Theory. The purchasing power parity (PPP) is the most enduring debate of literature in international macroeconomics. It is most controversial due to various puzzles and tested with different econometric models for certain group of countries. Therefore, the PPP is valid assumption while international comparison due to use of common exchange rate and the prevalence of Law of One price.

Design/Methodology/Approach: The validity of PPP for relative countries (Pakistan, China, Iran and Turkey) was tested and analyzed for the sample period 2001 to 2018 .

Findings: It is observed that exchange rates of Pakistan, China, Iran and Turkey are not consistent and constant. The deviations of PPP through structural changes identified and are not persistence over long period. Overall results reflected that there is an existence of long run equilibrium relation in between Pakistan and China as well as in between Iran and Turkey. The error correction model has confirmed the adjustment speed of short run disequilibrium to long term disequilibrium level. Implications/Originality/Value: The expected differential level of inflation has significant positive impact to exchange rate shift to Pakistan and trading activity patterns. The changes in foreign exchange market and commodity market due to economic integration are important implications for economic globalization.
\end{abstract}

\section{OPEN ACCESS}

(C) 2020 The authors, under a Creative Commons Attribution-Non

Commercial 4.0

Corresponding author's email address: shahid.imdad@yahoo.com

Recommended citation: Akash, R. S. I., Ghafoor, M. M., \& Ahmad, N., (2020). Testing the Validity of Purchasing Power Parity Theory and Dynamics of Exchange Rate Behavior (Pakistan, China, Iran and Turkey). Journal of Accounting and Finance in Emerging Economies, 6 (1), 127-144

DOI: $10.26710 /$ jafee.v6i1.1059

\section{Introduction}

The Law of one price indicates that the price of a commodity at domestic level should be the same at foreign level. However, the purchasing power parity theory is an economic theory which is based on underlying assumption of exchange rate and macro models in the open economy. It is a primary doctrine 
in the literature of international finance. The purchasing power theory is a comparative compression of money at that level where one currency per value measured at equal base to another currency. The value of home currency must have the equal value to foreign currency. Froot and Rogoff (1995), Sarno and Taylor (2002) studied the PPP theory which is found mixed for its current floating exchange rate period because of overvalued or undervalued of a currency.

The fluctuations in the exchange rate implicate movements towards or away from long time equilibrium level. It is very crucial for policy perspective. In accordance, law of one price holds that at a same time a money value of identical products should be same while purchase and sale among two economies. The economies have high degrees of economic interdependence and fluctuation in exchange rate. On the other hand, demand of one product is fluctuated due to shift in inflation of one economy. The change in demand from home country to foreign country will be continued until the change in currency value of foreign country appreciated. The PPP can have implications to explain that the real exchange rates found nonstationary.

The non-stationary of real exchange rate is being held. The theory describes that traditional PPP is failing to hold due to non-stationarity in the fundamentals economic. The economic fundamentals are the basic premise of equilibrium and a long term equilibrium level implicates to hold of PPP. A non validity of PPP should also have better implications for a wide set of subsequent theories of international finance. So, PPP is proposed to be as a valid proposition for best flow with reference to economic fundamentals.

Most of the international economic fundamentals based on flow of exchange rate. Therefore exchange rate may goes under the name of risks due to volatility for the long run. The phenomena of long standing puzzle may arise. The currency prices not at fundamental values are basic premise and quick adjustment may validate at any instant the prices at their fundamental values and short run disequilibrium. The phenomena will have a long standing puzzle of exchange rate. The puzzle of exchange rate may create misallocation of scarce resources. PPP can minimize the misallocation of scarce resources and help to create global prosperity. The inefficiency of quick adjustment may create the mispricing to lead the puzzle. The disequilibrium cause price distortion leads eventually international welfare losses. Thus it should be needed considerable effort to locate the exchange rates correctly priced or not excessively price and parity conditions hold. An empirical analysis can be used to help for exploration of real exchange rates stationarity. The stationarity level supported to co - integration analysis. It can be helpful for a system comprising to a nominal level of exchange rate, its foreign prices and domestic prices.

The previous literature supported that a non-linear trends is a key assumption to explain the failure of the theory. Furthermore, it should also assist to determine PPP may have been found valid in Pakistan, China, Iran and Turkey.

\section{Literature Review}

Khan and Qayyum (2007) identified the existence of PPP and validated a relationship of foreign exchange and integration of goods market. Chaung and Lai (1994) suggested supporting the assumption of purchasing power parity and indicating that two conditions are not consistent. Alba and Park (2005) showed that PPP holds under exchange rate to support for purchasing power parity theory. Bhatti (1996) examined that a nominal level of exchange rate is used to tend the domestic price ratio and foreign price that supports a real exchange rate behavior is also mean reversion. Taylor (2002) investigated the PPPP and found a favorable with floating exchange rates also linked to deviations due to purchasing power parity. Taylor and Sarno (1998) explored that a real dollar exchange rate during post Bretton Wood system found evidence of mean reverting behavior of PPP. Sercu et.al (1995) observed the behavior of exchange rate and consider the inter dependence and non-traded goods of economy. Arize et.al (2004) suggested about the purchasing power parity assumptions holds with the conditions of equilibrium and found PPP more stable in Asia as in African. Murray and Papell (2004) concluded that Taylor was very 
strong in study to perfume the unit root test of lags selection and rejected favor the assumption of PPP. Elsadig (2011) tested PPP of US dollar and Japanese Yen. A penal unit root and cointegration tests employed and mixed results found regarded to existence of PPP. Chortareas and Kapetanios (2013) studied the relationship in prices and exchange rates under purchasing power parity theory of MIST (Mexico, Indonesia, South Koreas and Turkey) and BRICS (Brazil, Russian, Indian, China, and South African) countries. Glenville (2013) also explained about the exchange rate and price level integration of bivariate countries. The exchange rate stationarity proved and found a long term positive relation among price levels and exchange rates exist. Kamal (2014) explored an empirical study of real exchange rate of short run and long run relation to buying power. The unit root test does not support PPP due to non stationarity of exchange rate and no cointegration exists between price and exchange rate. Zyoud (2015) tested the PPP and found existence but weak level of PPP in short term due to slow movement as compared to change in value of currency. Bhatti (1996) and Shively (2001) supported a long term existence, relative price level move with the proportionate of nominal exchange rate risk and real exchange rate with its equilibrium level in long term. Shively (2001) found PPP have no impact on real exchange rate. However, PPP play a vital role to determine the nominal exchange rate. Kanyembo and Sheefeni (2013) indicated that the PPP holds and exist in the long run between South Africa and Zambia. He et al. (2015) suggested the globalization under the world economy. The China's foreign trade and its role with exchange rate and increase in inflation to its economy. Dixon et al. (2016) provided about the exchange rate reforms of specific regime announced by China to make flexible exchange rate economy. It also considers the monetary policy to develop industry and economy. Ma et al. (2017) argued the validity of PPP which have no strong base in China, Korea and Japan But also found that PPP holds and exist in China under the quantile approach specifically. The heterogeneity and non-stationarity found in disorder due to PPP deviated and found a long run rapid fluctuation in exchange rate. The long run parity related to Pakistan and India, PPP holds. The previous literature tested only existence of long term relationship but not short term relationship of PPP and exchange rate. This research hypothesis explored that whether the long term and short term relationship of PPP and dynamics of exchange rate exist or not.

\section{Data and Methodology}

\subsection{Data}

The validity of PPP for Pakistan, China, Iran and Turkey is tested and analyzed for the sample period January 1, 2001 to December 31, 2018. PPP theory can be viewed under the strong foundation of law of one price. The difference in transaction cost such as transportation cost, tax and tariffs are the basic premise to violate the law of one price. Actually, the process of arbitrage will be used to ensure that same good traded in world market, would be sold at same price in every country. The prices should shows at similar price in a common currency.

The $\mathrm{P}_{\mathrm{t}}$ and $\mathrm{SP}_{\mathrm{t}}$ * are the domestic and foreign prices of currency for goods or services. Thus, the law of one price in term of exchange rate prices:

$\mathrm{P}_{\mathrm{t}}=\mathrm{e} \mathrm{SP_{ \textrm {t } }} *$

To make a comparative relation to domestic and foreign currencies of countries should be as under:

$$
\begin{aligned}
& \mathrm{Pt}=\mathrm{eSPt}^{*} \\
& \mathrm{E}=\mathrm{Pt} / \mathrm{SPt}^{*}
\end{aligned}
$$

The theory of exchange rates, PPP equation (2) predicted the exchange rate to adjust at same level of price. The absolute PPP underlying assumption of real exchange rate and nominal exchange rate should also be adjusted as under:

$$
\frac{e S P t *}{P t}=1
$$


Where Qt is the real exchange rate can be as under:

$$
Q t=\frac{e S P t *}{P t}=1
$$

PPP theory is established by Cassel (1916) that a floating exchange rate and nominal exchange rate cannot be deviated from its PPP. There should not be any transaction cost such as trade restrictions and transportation cost. Assuming that there is the LOP hold as under

$$
\sum_{j=1}^{n} W_{j} P t_{j}=\operatorname{es} S \sum_{j=1}^{n} W *_{j} P t *_{j}
$$

PPP theory tests are used to analyze the stationarity of exchange rate and price level series related to two countries. The unit root test applied. Pt as prices at domestic level and SPt* as prices at foreign level of other countries to compare with Pakistan. The real exchange rate in the logarithm formation and Qt, the real exchange rate should be calculated as:

$\log (\mathrm{Qt})=\log (\mathrm{e})+\log \left(\mathrm{SPt}^{*}\right)-\log (\mathrm{Pt})$

Augmented Dickey Fuller (ADF) (1979) unit root test for simple autoregressive model (AR) is as follows.

$$
\mathrm{Xt}=\Delta \mathrm{Xt}-1+\mu \mathrm{t}
$$

$\mathrm{Xt}$ is a variable for the time $\mathrm{t}, \Delta$ is a coefficient and $\mu \mathrm{t}$ is an error term. $\Delta \mathrm{Xt}$ at 1 st difference or second difference.

$$
\Delta \mathrm{Xt}=(\Delta-1) \mathrm{Xt}-1+\mu \mathrm{t}=\lambda \mathrm{Xt}-1+\mu \mathrm{t}
$$

The Phillip Perron (PP) (1988) test in autoregressive (AR) model for unit root test as under applied.

$$
\mathrm{Xt}=\lambda 0+\lambda 1 \mathrm{Xt}-1+\lambda 2 t\left[t-\frac{T}{2}\right]+\mu \mathrm{t}
$$

The Johansen $(1988 ; 1991)$ and Johansen-Juselius (1990) test is a technique to identify cointegration.

$$
\Delta \mathrm{Xt}=\alpha+\sum_{\mathrm{t}=1}^{\mathrm{n}-1} \gamma \mathrm{t} \Delta \mathrm{Xt}-1+\alpha \mathrm{t} \Delta \mathrm{Xt}-1+\mu \mathrm{t}
$$

$\alpha$ in an equation of relationship is being a constant, $\mathrm{Xt}$ is a matrix of vectors, $\gamma$ and $\alpha$ are the parameters or coefficients. $\Delta$ is taken as a operator of change for vectors in the matrix. Trace statistics equation is as under.

$$
\dot{Y}_{\text {trace }}=-\mathrm{T} \sum \operatorname{nlog}(1-\gamma \mathrm{Q})
$$

The maximum Eigen value can have a prove of hypothesis of cointegration exist due to more than its critical value.

$$
\dot{Y}_{\text {Maximum }}=-\mathrm{T} \operatorname{nlog}(1-\lambda \mathrm{P}+1)
$$

$\lambda \mathrm{P}+1, \ldots \ldots \ldots, \lambda \mathrm{P}=(\mathrm{N}-\mathrm{P})$ is being used to its small square; where the $\mathrm{T}$ is taken a level of observations at its area specified. Bivariate co-integration analysis is used to observe a long term relationship among two series. Bivariate autoregressive process among two different series identifying co-integration to show its effects in the long term. 


$$
\begin{aligned}
& \mathrm{X}=\alpha_{0}+\sum_{\mathrm{i}=1}^{\mathrm{n}} \lambda_{i} X_{t-1}+\sum_{\mathrm{i}=1}^{\mathrm{n}} \delta C_{t-1}+\mu_{t} \\
& \mathrm{Y}_{t}=\alpha_{0}+\sum_{\mathrm{i}=1}^{\mathrm{n}} \lambda_{i} Y_{t-1}+\sum_{\mathrm{i}=1}^{\mathrm{n}} f_{i} \lambda_{t-1}+\mu_{t}
\end{aligned}
$$

$\mathrm{X}_{\mathrm{t}}$ and $\mathrm{Y}_{\mathrm{t}}$ are the series of stationarity, $\alpha$ is a constant.

$$
\mathrm{Y}_{\mathrm{t}}=\alpha_{0}+\sum_{\mathrm{i}=1}^{\mathrm{n}} \lambda_{\mathrm{i}} \mathrm{Y}_{\mathrm{t}-1}+\sum_{\mathrm{i}=1}^{\mathrm{n}} \Delta_{\mathrm{i}} \mathrm{Y}_{\mathrm{t}-1}+\mu_{\mathrm{t}}
$$

The Eagle Granger causality analysis test is employed to identify the cause and effect whether one time series has lead and lag relationship to another. Therefore, a Granger cause is useful and assists to determine the factor of causality relation and lead lag values selection process.

$$
\delta_{\text {prob }}\left(X_{t+n} \mid €_{t}=\delta_{\text {prob }}\left(X_{t+n} \mid \mu_{t}\right)\right.
$$

A conditional probability $\mathrm{P}$ probability set of information $\mathrm{Xt}$ at time $\mathrm{t}$ on $\mathrm{Xt}+\mathrm{n}$ values at past and information set $\mu_{t}$ values contained for both Xt and Yt for the given period of time. Vector error model of correction is being used to identify misspecification to evaluate the relation at short term and adjustments at short term with reference to recent economic activity.

$$
\begin{aligned}
& \Delta \mathrm{X}_{\mathrm{t}}=\lambda+\sum_{\mathrm{i}=1}^{\mathrm{n}} \lambda_{\mathrm{i}} \Delta \mathrm{X}_{\mathrm{t}-\mathrm{i}}+\sum_{\mathrm{i}=1}^{\mathrm{n}} \delta_{\mathrm{i}} \Delta \mathrm{X}_{\mathrm{t}-1}+\gamma \mathrm{R}_{\mathrm{i}-1}+\mu_{\mathrm{t}} \\
& \Delta \mathrm{Y}_{\mathrm{t}}=\lambda+\sum_{\mathrm{i}=1}^{\mathrm{n}} \lambda_{\mathrm{i}} \mathrm{Y}_{\mathrm{t}-\mathrm{i}}+\sum_{\mathrm{i}=1}^{\mathrm{n}} \delta \Delta \lambda_{\mathrm{t}-1}+£ \mathrm{R}_{\mathrm{i}-1}+\mu_{\mathrm{t}}
\end{aligned}
$$

\begin{tabular}{|c|c|c|c|c|}
\hline Variables & $\begin{array}{c}\text { ADF } \\
\text { Test at } \\
\text { Level 5\% }\end{array}$ & $\begin{array}{c}\text { ADF } \\
\text { Test at } 1^{\text {st }} \\
\text { difference }\end{array}$ & $\begin{array}{c}\text { PP } \\
\text { Test at Level } \\
5 \% \\
\end{array}$ & $\begin{array}{c}\text { PP } \\
\text { Test at } 1^{\text {st }} \\
\text { difference }\end{array}$ \\
\hline \multicolumn{5}{|l|}{$\begin{array}{l}\text { Pakistan Vs. China } \\
\text { Parameters }\end{array}$} \\
\hline Cpi_Pk & -1.6464 & -7.1817 & 6.2456 & -8.6081 \\
\hline Cpi_China & -1.3456 & 3.2346 & 5.4078 & -8.3424 \\
\hline S_Pk_China & 1.2434 & -8.5678 & 2.5679 & -9.3657 \\
\hline Q_Pk_China & -2.4567 & -10.4327 & -4.8970 & -17.5674 \\
\hline Rp_Pk_China & -3.4367 & -11.0987 & -7.7689 & -23.8970 \\
\hline \multicolumn{5}{|l|}{$\begin{array}{l}\text { Pakistan Vs. Iran } \\
\text { Parameters }\end{array}$} \\
\hline Cpi_Pk & -1.1235 & -7.1289 & 5.5643 & -6.6754 \\
\hline Cpi_Iran & 2.4536 & -3.6754 & 3.5609 & -7.2360 \\
\hline S_Pk_Iran & -1.1137 & -12.8970 & -1.1872 & -12.896 \\
\hline Q_Pk_Iran & -2.6754 & -11.3450 & -2.2345 & -10.5490 \\
\hline Rp_Pk_Iran & -2.3809 & -9.4980 & -5.9087 & -7.5789 \\
\hline \multicolumn{5}{|l|}{$\begin{array}{l}\text { Pakistan Vs. Turkey } \\
\text { Parameters }\end{array}$} \\
\hline Cpi_Pk & -1.3478 & -7.1618 & 5.9086 & -8.6789 \\
\hline Cpi_Turkey & 3.4678 & -5.6790 & 3.7890 & -9.1980 \\
\hline
\end{tabular}

The stationary series are taken as $\mathrm{Xt}$ and $\mathrm{Yt}$ in the given equations. $\lambda$ is constants. $\mu_{\mathrm{t}}$ is an term of error and $\mathrm{Ri}$ is term of correction, $A_{i}$ and $B_{i}, \delta$, f, are parameters or coefficients. $\gamma, \theta$ are parameters or coefficients of error, positive integer value $n$ and number of values $i$ is taken.

\section{Results and Discussion:}

Table - 1 ADF and PP Tests 


\begin{tabular}{|l|r|r|r|r|}
\hline S_Pk_Turkey & -1.0345 & -8.6790 & -1.3478 & -8.9807 \\
\hline Q_Pk_Turkey & 1.6780 & -7.5697 & 1.7098 & -9.3467 \\
\hline Rp_Pk_Turkey & 1.0987 & -8.7698 & 1.8907 & -9.7854 \\
\hline
\end{tabular}

Table 3.1: Critical Values:

\begin{tabular}{|l|c|c|c|}
\hline Probabilities & $\mathbf{1 \%}$ & $\mathbf{5 \%}$ & $\mathbf{1 0 \%}$ \\
\hline ADF at Level & -3.43228 & -2.86228 & -2.56721 \\
\hline ADF at $\mathbf{1}^{\text {st }}$ difference & -3.46446 & -2.87644 & -2.57479 \\
\hline PP at Level & -3.43228 & -2.86228 & -2.56721 \\
\hline PP at1 $^{\text {st }}$ difference & -3.46446 & -2.87644 & -2.57479 \\
\hline
\end{tabular}

Table 1 indicates that ADF and PP tests are applied to test the stationarity of data. The series is to be considered stationary when there exist no trend. The series of data tested at first order difference and second order difference when data is not stationary at level. The above results indicated that the series became stationary at $1^{\text {st }}$ difference for Pakistan, China, Iran and Turkey. Co-integration tests are used in determining the long run relationship among the series. The ADF and PP tests indicate that the series of data become stationary at first order difference. The prove of data is stationary is a pre-requisite to apply Johansen and Juselius co-integration tests. The selection of series statistics at lag length is made according to SIC - Schwarz Information Criterion. The cointegration model is assumed constant and linear trend. Table 2 indicates that there exist long term relationships due to cointegration factors exist.

Table 2: Johanson Multivariate Cointegration of Pakistan, China, Iran and Turkey (Trace Statistics)

\begin{tabular}{|c|c|c|c|c|c|}
\hline Variables & $\begin{array}{l}\text { Hypothe } \\
\text { sis } \\
\text { No. of } \\
\text { CE(s) }\end{array}$ & $\begin{array}{l}\text { Eigen } \\
\text { Value }\end{array}$ & $\begin{array}{l}\text { Trace } \\
\text { Statistic }\end{array}$ & $\begin{array}{l}\text { At } 5 \% \\
\text { Critical } \\
\text { Value }\end{array}$ & Critical Remarks \\
\hline \multicolumn{5}{|c|}{ Pakistan Vs. China Parameters } & \multirow{6}{*}{$\begin{array}{l}\text { In this there exist } 5 \text { Co- } \\
\text { Integration Vector at Critical } \\
\text { level of } 5 \% \text {. }\end{array}$} \\
\hline Cpi_Pk & None $*$ & $\begin{array}{r}185.51 \\
4\end{array}$ & 69.818 & 0 & \\
\hline Cpi_China & $\begin{array}{l}\text { At most } 1 \\
*\end{array}$ & $\begin{array}{r}104.92 \\
4\end{array}$ & 47.856 & 0 & \\
\hline S_Pk_China & $\begin{array}{l}\text { At most } 2 \\
*\end{array}$ & 62.312 & 29.797 & 0 & \\
\hline $\begin{array}{l}\text { Q_Pk_Chin } \\
\text { a }\end{array}$ & $\begin{array}{l}\text { At most } 3 \\
*\end{array}$ & 35.156 & 15.494 & 0 & \\
\hline $\begin{array}{l}\text { Rp_Pk_Chi } \\
\text { na }\end{array}$ & $\begin{array}{l}\text { At most } 4 \\
*\end{array}$ & $\begin{array}{r}10.256 \\
8\end{array}$ & 3.8414 & 0 & \\
\hline \multicolumn{5}{|c|}{ Pakistan Vs. Iran Parameters. } & \multirow{6}{*}{$\begin{array}{l}\text { In this there exist } 4 \text { Co- } \\
\text { Integration Vector at Critical } \\
\text { level of } 5 \% \text {. }\end{array}$} \\
\hline Cpi_Pk & None * & $\begin{array}{r}185.03 \\
6 \\
\end{array}$ & 69.818 & 0 & \\
\hline Cpi_Iran & $\begin{array}{l}\text { At most } 1 \\
*\end{array}$ & $\begin{array}{r}118.28 \\
9 \\
\end{array}$ & 47.856 & 0 & \\
\hline S_Pk_Iran & $\begin{array}{l}\text { At most } 2 \\
*\end{array}$ & $\begin{array}{r}72.445 \\
1 \\
\end{array}$ & 29.797 & 0 & \\
\hline Q_Pk_Iran & $\begin{array}{l}\text { At most } 3 \\
*\end{array}$ & $\begin{array}{r}34.982 \\
9 \\
\end{array}$ & 15.494 & 0 & \\
\hline Rp_Pk_Iran & $\begin{array}{l}\text { At most } 4 \\
*\end{array}$ & 0.050 & 3.8414 & 0.8229 & \\
\hline \multicolumn{5}{|c|}{ Pakistan Vs. Turkey Parameters } & In this there exist 4 Co- \\
\hline
\end{tabular}




\begin{tabular}{|c|c|c|c|c|c|}
\hline Cpi_Pk & None * & $\begin{array}{r}232.63 \\
5\end{array}$ & 69.818 & 0 & \multirow[t]{5}{*}{$\begin{array}{l}\text { Integration Vector at Critical } \\
\text { level of } 5 \% \text {. }\end{array}$} \\
\hline Cpi_Turkey & $\begin{array}{l}\text { At most } 1 \\
*\end{array}$ & $\begin{array}{r}160.36 \\
5\end{array}$ & 47.8561 & 0 & \\
\hline $\begin{array}{l}\text { S_Pk_ } \\
\text { Turkey }\end{array}$ & $\begin{array}{l}\text { At most } 2 \\
*\end{array}$ & 99.930 & 29.7970 & 0 & \\
\hline $\begin{array}{l}\text { Q_Pk_ } \\
\text { Turkey }\end{array}$ & $\begin{array}{l}\text { At most } 3 \\
*\end{array}$ & 44.448 & 15.494 & 0 & \\
\hline $\begin{array}{l}\text { Rp_Pk_ } \\
\text { Turkey }\end{array}$ & $\begin{array}{l}\text { At most } 4 \\
*\end{array}$ & 1.382 & 3.841 & 0.2396 & \\
\hline
\end{tabular}

There exist 5 Co-integrating vectors exist between Pakistan and China which indicates that long relationship exist between these two economies. However there exist 5 co-integrating vectors exist among Pakistan and china as well which also elaborate existence of long run relationships. Moreover there also exist 5 co-integrating vectors among Pakistan and Turkey as well. The trace statistics accepts $H_{0}$ and rejects $H_{1}$. The Maximum Eigen Value tests also applied to confirm the presence of long run relationship. Table 3 indicates the results regarding Maximum Eigen Value.

Table 3: Johanson Multivariate Cointegration of Pakistan, China, Iran and Turkey (Maximum Eigen Value)

\begin{tabular}{|c|c|c|c|c|c|}
\hline $\begin{array}{c}\text { Equity } \\
\text { Markets. }\end{array}$ & $\begin{array}{l}\text { Hypothe } \\
\text { sis } \\
\text { No. of } \\
\text { CE(s) }\end{array}$ & $\begin{array}{l}\text { Eigen } \\
\text { Value }\end{array}$ & $\begin{array}{l}\text { Max. Eigen } \\
\text { Value } \\
\text { Statistics. }\end{array}$ & $\begin{array}{l}5 \% \\
\text { Critical } \\
\text { Value }\end{array}$ & Critical Remarks \\
\hline \multicolumn{5}{|c|}{ Pakistan Vs. China Parameters } & \multirow{6}{*}{$\begin{array}{l}\text { In this there exist } 5 \text { Co- } \\
\text { Integration Vector at Critical } \\
\text { level of } 5 \% \text {. }\end{array}$} \\
\hline Cpi_Pk & None * & 80.589 & 33.876 & 0 & \\
\hline Cpi_China & $\begin{array}{l}\text { At most } 1 \\
*\end{array}$ & 42.612 & 27.584 & 0 & \\
\hline S_Pk_China & $\begin{array}{l}\text { At most } 2 \\
*\end{array}$ & 27.155 & 21.131 & 0 & \\
\hline $\begin{array}{l}\text { Q_Pk_Chin } \\
\text { a }\end{array}$ & $\begin{array}{l}\text { At most } 3 \\
*\end{array}$ & 24.900 & 14.264 & 0 & \\
\hline $\begin{array}{l}\text { Rp_Pk_Chi } \\
\text { na }\end{array}$ & $\begin{array}{l}\text { At most } 4 \\
*\end{array}$ & 10.256 & 3.841 & 0 & \\
\hline \multicolumn{5}{|c|}{ Pakistan Vs. Iran Parameters. } & \multirow{6}{*}{$\begin{array}{l}\text { In this there exist } 4 \text { Co- } \\
\text { Integration Vector at Critical } \\
\text { level of 5\%. }\end{array}$} \\
\hline Cpi_Pk & None * & 66.746 & 33.876 & 0 & \\
\hline Cpi_Iran & $\begin{array}{l}\text { At most } 1 \\
*\end{array}$ & 45.844 & 27.584 & 0 & \\
\hline S_Pk_Iran & $\begin{array}{l}\text { At most } 2 \\
*\end{array}$ & 37.462 & 21.131 & 0 & \\
\hline Q_Pk_Iran & $\begin{array}{l}\text { At most } 3 \\
*\end{array}$ & 34.932 & 14.264 & 0 & \\
\hline Rp_Pk_Iran & $\begin{array}{l}\text { At most } 4 \\
*\end{array}$ & 0.0500 & 3.841 & 0.822 & \\
\hline \multicolumn{5}{|c|}{ Pakistan Vs. Turkey Parameters } & \multirow{3}{*}{$\begin{array}{l}\text { In this there exist } 4 \text { Co- } \\
\text { Integration Vector at Critical } \\
\text { level of } 5 \% \text {. }\end{array}$} \\
\hline Cpi_Pk & None * & 72.270 & 33.876 & 0 & \\
\hline Cpi_Turkey & $\begin{array}{l}\text { At most } 1 \\
*\end{array}$ & 60.434 & 27.584 & 0 & \\
\hline
\end{tabular}




\begin{tabular}{|l|l|r|r|r|}
\hline $\begin{array}{l}\text { S_Pk_ } \\
\text { Turkey }\end{array}$ & $\begin{array}{l}\text { At most } 2 \\
*\end{array}$ & 55.482 & 21.131 & 0 \\
\hline $\begin{array}{l}\text { Q_Pk_ } \\
\text { Turkey }\end{array}$ & $\begin{array}{l}\text { At most } 3 \\
*\end{array}$ & 43.065 & 14.264 & 0 \\
\hline $\begin{array}{l}\text { Rp_Pk_ } \\
\text { Turkey }\end{array}$ & $\begin{array}{l}\text { At most } 4 \\
*\end{array}$ & 1.382 & 3.841 & 0.2396 \\
\hline
\end{tabular}

According to Table 3 again there exist 5 Co-integrating vectors exist between Pakistan and China which indicates that long relationship exist between these two economies. However there exist 5 co-integrating vectors exist among Pakistan and china as well which also elaborate existence of long run relationships. Moreover there also exist 5 co-integrating vectors among Pakistan and Turkey as well. The Maximum Eigen value test also accepts $H_{0}$ and rejects $H_{1}$. Table 4 a. describes bivariate co-integration to explore the long run relationship among the purchasing power parity variables of Pakistan and China.

Table 4 a. Bivariate Cointegration for Pakistan Vs. China:

\begin{tabular}{|c|c|c|c|c|c|}
\hline Equity Markets. & $\begin{array}{l}\text { Hypoth } \\
\text { esis }\end{array}$ & $\begin{array}{c}\text { Eige } \\
\text { n } \\
\text { Valu } \\
\text { e }\end{array}$ & $\begin{array}{c}\text { Trace } \\
\text { Statisti } \\
\text { cs. }\end{array}$ & $\begin{array}{l}\text { At 5\% } \\
\text { Critical } \\
\text { Value }\end{array}$ & Critical Remarks. \\
\hline \multicolumn{6}{|c|}{ Pakistan Vs. China Parameters. } \\
\hline Cpi_China --- Cpi_Pk & $\begin{array}{l}\text { None } * \\
\text { At most } \\
1 *\end{array}$ & $\begin{array}{r}0.32 \\
9\end{array}$ & 22.399 & 13.324 & Co-integration Exist. \\
\hline $\begin{array}{l}\text { Cpi_China --- } \\
\text { Q_Pk_China }\end{array}$ & $\begin{array}{l}\text { None } \\
\text { At most } \\
1\end{array}$ & $\begin{array}{r}0.06 \\
5\end{array}$ & 0.709 & 4.598 & Co-integration not Exist. \\
\hline $\begin{array}{l}\text { Cpi_China --- } \\
\text { Rp_Pk_China }\end{array}$ & $\begin{array}{l}\text { None } * \\
\text { At most } \\
1 *\end{array}$ & $\begin{array}{r}0.09 \\
8\end{array}$ & 14.678 & 12.654 & Co-integration Exist. \\
\hline $\begin{array}{l}\text { Cpi_China --- } \\
\text { S_Pk_China }\end{array}$ & $\begin{array}{l}\text { None } * \\
\text { At most } \\
1 *\end{array}$ & $\begin{array}{r}0.00 \\
0\end{array}$ & 0.0760 & 4.325 & Co-integration not Exist. \\
\hline Cpi_Pk --- Q_Pk_China & $\begin{array}{l}\text { None }{ }^{*} \\
\text { At most } \\
1 *\end{array}$ & $\begin{array}{r}0.43 \\
2\end{array}$ & 33.098 & 19.398 & Co-integration Exist. \\
\hline $\begin{array}{l}\text { Cpi_Pk --- } \\
\text { Rp_Pk_China }\end{array}$ & $\begin{array}{l}\text { None } * \\
\text { At most } \\
1 *\end{array}$ & $\begin{array}{r}0.54 \\
6\end{array}$ & 0.598 & 2.629 & Co-integration Exist. \\
\hline Cpi_Pk --- S_Pk_China & $\begin{array}{l}\text { None } \\
\text { At most } \\
1\end{array}$ & $\begin{array}{r}0.98 \\
2\end{array}$ & 6.294 & 13.271 & Co-integration not Exist. \\
\hline $\begin{array}{l}\text { S_Pk_China --- } \\
\text { Q_Pk_China }\end{array}$ & $\begin{array}{l}\text { None } \\
\text { At most } \\
1\end{array}$ & $\begin{array}{r}0.59 \\
1\end{array}$ & 0.762 & 4.145 & Co-integration not Exist. \\
\hline
\end{tabular}

The above results indicate that there exist long run relationship between CPI, Relative Price, Real and Nominal exchange rates of Pakistan and China. Table 4 b. describes bivariate co-integration to explore the long run relationship among the purchasing power parity variables of Pakistan and Iran.

Table 4 b. Bivariate Cointegration for Pakistan Vs. Iran: 


\begin{tabular}{|c|c|c|c|c|c|}
\hline Equity Markets. & $\begin{array}{l}\text { Hypothe } \\
\text { sis }\end{array}$ & $\begin{array}{c}\text { Eige } \\
\text { n } \\
\text { Valu } \\
\text { e }\end{array}$ & $\begin{array}{c}\text { Trace } \\
\text { Statisti } \\
\text { cs. }\end{array}$ & $\begin{array}{c}\text { At 5\% } \\
\text { Critical } \\
\text { Value }\end{array}$ & Critical Remarks. \\
\hline \multicolumn{6}{|c|}{ Pakistan Vs. Iran Parameters. } \\
\hline Cpi_Iran --- Cpi_Pk & $\begin{array}{l}\text { None } \\
\text { At most } \\
1\end{array}$ & $\begin{array}{r}0.60 \\
9\end{array}$ & 11.940 & 16.109 & $\begin{array}{l}\text { Co-integration not } \\
\text { Exist. }\end{array}$ \\
\hline Cpi_Iran --- Q_Pk_Iran & $\begin{array}{l}\text { None } \\
\text { At most } \\
1\end{array}$ & $\begin{array}{r}0.90 \\
8\end{array}$ & 0.419 & 2.932 & $\begin{array}{l}\text { Co-integration not } \\
\text { Exist. }\end{array}$ \\
\hline Cpi_Iran --- Rp_Pk_Iran & $\begin{array}{l}\text { None } \\
\text { At most } \\
1\end{array}$ & $\begin{array}{r}0.18 \\
5\end{array}$ & 9.281 & 14.286 & $\begin{array}{l}\text { Co-integration not } \\
\text { Exist. }\end{array}$ \\
\hline Cpi_Iran --- S_Pk_Iran & $\begin{array}{l}\text { None } \\
\text { At most } \\
1\end{array}$ & $\begin{array}{r}0.00 \\
1\end{array}$ & 0.742 & 4.263 & $\begin{array}{l}\text { Co-integration not } \\
\text { Exist. }\end{array}$ \\
\hline Cpi_Pk --- Q_Pk_Iran & $\begin{array}{l}\text { None } \\
\text { At most } \\
1\end{array}$ & $\begin{array}{r}0.26 \\
1\end{array}$ & 13.870 & 17.797 & $\begin{array}{l}\text { Co-integration not } \\
\text { Exist. }\end{array}$ \\
\hline Cpi_Pk --- Rp_Pk_Iran & $\begin{array}{l}\text { None } \\
\text { At most } \\
1\end{array}$ & $\begin{array}{r}0.00 \\
6\end{array}$ & 0.967 & 3.761 & $\begin{array}{l}\text { Co-integration not } \\
\text { Exist. }\end{array}$ \\
\hline Cpi_Pk --- S_Pk_Iran & $\begin{array}{l}\text { None } * \\
\text { At most } \\
1\end{array}$ & $\begin{array}{r}0.09 \\
8\end{array}$ & 6.173 & 18.4947 & $\begin{array}{l}\text { Co-integration one } \\
\text { Exist. }\end{array}$ \\
\hline S_Pk_Iran--- Q_Pk_Iran & $\begin{array}{l}\text { None } * \\
\text { At most } \\
1\end{array}$ & $\begin{array}{r}0.79 \\
1\end{array}$ & 0.485 & 4.018 & $\begin{array}{l}\text { Co-integration one } \\
\text { Exist. }\end{array}$ \\
\hline
\end{tabular}

The above results indicate that there exist no long run relationship between CPI, Relative Price, Real and Nominal exchange rates of Pakistan and Iran. Table $4 \mathrm{c}$ describes a bivariate co-integration to explore the long run relationship between the purchasing power parity variables of Pakistan and Turkey.

Table 4 c. Bivariate Cointegration for Pakistan Vs. Turkey:

\begin{tabular}{|c|c|c|c|c|c|}
\hline Equity Markets. & $\begin{array}{l}\text { Hypoth } \\
\text { esis }\end{array}$ & $\begin{array}{c}\text { Eige } \\
\text { n } \\
\text { Valu } \\
\text { e }\end{array}$ & $\begin{array}{c}\text { Trace } \\
\text { Statist } \\
\text { ics }\end{array}$ & $\begin{array}{c}\text { At 5\% } \\
\text { Critical } \\
\text { Value }\end{array}$ & Critical Remarks. \\
\hline \multicolumn{6}{|c|}{ Pakistan Vs. Turkey Parameters. } \\
\hline Cpi_Turkey --- Cpi_Pk & $\begin{array}{l}\text { None * } \\
\text { At most } \\
1\end{array}$ & $\begin{array}{r}0.49 \\
2\end{array}$ & 19.481 & 14.086 & $\begin{array}{l}\text { Co-integration one } \\
\text { Exist. }\end{array}$ \\
\hline Cpi_Turkey --- Q_Pk_Turkey & $\begin{array}{l}\text { None } * \\
\text { At most } \\
1 *\end{array}$ & $\begin{array}{r}0.83 \\
0\end{array}$ & 0.963 & 4.198 & $\begin{array}{c}\text { Co-integration one } \\
\text { Exist. }\end{array}$ \\
\hline Cpi_Turkey --- Rp_Pk_Turkey & $\begin{array}{l}\text { None } * \\
\text { At most } \\
1 *\end{array}$ & $\begin{array}{r}0.05 \\
6\end{array}$ & 16.904 & 13.648 & $\begin{array}{c}\text { Co-integration one } \\
\text { Exist. }\end{array}$ \\
\hline
\end{tabular}




\begin{tabular}{|l|l|r|r|r|c|}
\hline Cpi_Turkey --- S_Pk_Turkey & $\begin{array}{l}\text { None } \\
\text { At most } \\
1\end{array}$ & $\begin{array}{r}0.04 \\
5\end{array}$ & 0.095 & 4.674 & $\begin{array}{c}\text { Co-integration not } \\
\text { Exist. }\end{array}$ \\
\hline Cpi_Pk --- Q_Pk_Turkey & $\begin{array}{l}\text { None } \\
\text { At most } \\
1\end{array}$ & $\begin{array}{r}0.00 \\
9\end{array}$ & 13.969 & 18.739 & $\begin{array}{c}\text { Co-integration not } \\
\text { Exist. }\end{array}$ \\
\hline Cpi_Pk --- Rp_Pk_Turkey & $\begin{array}{l}\text { None } * \\
\text { At most } \\
1\end{array}$ & $\begin{array}{r}0.05 \\
9\end{array}$ & 8.765 & 5.512 & $\begin{array}{c}\text { Co-integration one } \\
\text { Exist. }\end{array}$ \\
\hline Cpi_Pk --- S_Pk_Turkey & $\begin{array}{l}\text { None } * \\
\text { At most } \\
1 *\end{array}$ & $\begin{array}{r}0.85 \\
4\end{array}$ & 12.281 & 9.837 & $\begin{array}{c}\text { Co-integration one } \\
\text { Exist. }\end{array}$ \\
\hline S_Pk_Turkey --- Q_Pk_Turkey & $\begin{array}{l}\text { None } * \\
\text { At most } \\
1 *\end{array}$ & $\begin{array}{r}0.93 \\
8\end{array}$ & 2.164 & 1.419 & $\begin{array}{c}\text { Co-integration one } \\
\text { Exist. }\end{array}$ \\
\hline
\end{tabular}

The above results indicate that there exist no long run relationship between CPI, Relative Price, Real and Nominal exchange rates of Pakistan and Turkey. There is no lead lag relationship in between the PPP variables of Pakistan and China. if $H_{0}$ where $\mathrm{p}>0.05$ then the null hypothesis accepted. $H_{0}$ where $\mathrm{p}>$ 0.05. So, hypothesis accepted. Table 5 a. indicates that changes of Cpi of China lead lag and causes of Cpi of Pakistan, real exchange rate, nominal exchange rate and relative prices.

Table 5 a.: Pair wise Pakistan versus China Causality Test:

\begin{tabular}{|c|c|c|}
\hline Null Hypothesis: & F-Statistic & Probability \\
\hline $\begin{array}{l}\text { CPI_CHI_RT } \\
\text { CPI_PK_RT }\end{array}$ & 2.44384 & $0.0896 * * *$ \\
\hline $\begin{array}{l}\text { CPI_PK_RT } \\
\text { CPI_CHI_RT }\end{array}$ & 1.71505 & 0.1828 \\
\hline $\begin{array}{l}\text { Q_RT }=- \\
\text { CPI_PK_RT }\end{array}$ & 1.88586 & 0.1546 \\
\hline CPI_PK_RT & 0.70432 & 0.4958 \\
\hline $\begin{array}{l}\text { RP_RT } \longrightarrow \\
\text { CPI_PK_RT }\end{array}$ & 3.88032 & $0.0224 * *$ \\
\hline $\begin{array}{l}\text { CPI_PK_RT } \\
\text { RP_RT }\end{array}$ & 0.52225 & 0.5941 \\
\hline $\begin{array}{l}\text { S_RT } \\
\text { CPI_PK_RT }\end{array}$ & 1.03235 & 0.3582 \\
\hline CPI_PK_RT $\longrightarrow$ & 0.61005 & 0.5444 \\
\hline $\begin{array}{l}\text { Q_RT } \\
\text { CPI_CHI_RT }\end{array}$ & 0.16002 & 0.8522 \\
\hline CPI_CHI_RT & 1.05499 & 0.3503 \\
\hline $\begin{array}{l}\text { RP_RT } \longrightarrow \\
\text { CPI_CHI_RT }\end{array}$ & 0.82217 & 0.4411 \\
\hline $\begin{array}{l}\text { CPI_CHI_RT } \\
\text { RP_RT }\end{array}$ & 2.91958 & $0.0565^{* * * *}$ \\
\hline $\begin{array}{l}\text { S_RT } \\
\text { CPI_CHI_RT }\end{array}$ & 0.08241 & 0.9209 \\
\hline CPI_CHI_RT $\longrightarrow$ & 0.17878 & 0.8364 \\
\hline RP_RT $\longrightarrow$ & 2.42344 & $0.0914 * * *$ \\
\hline
\end{tabular}




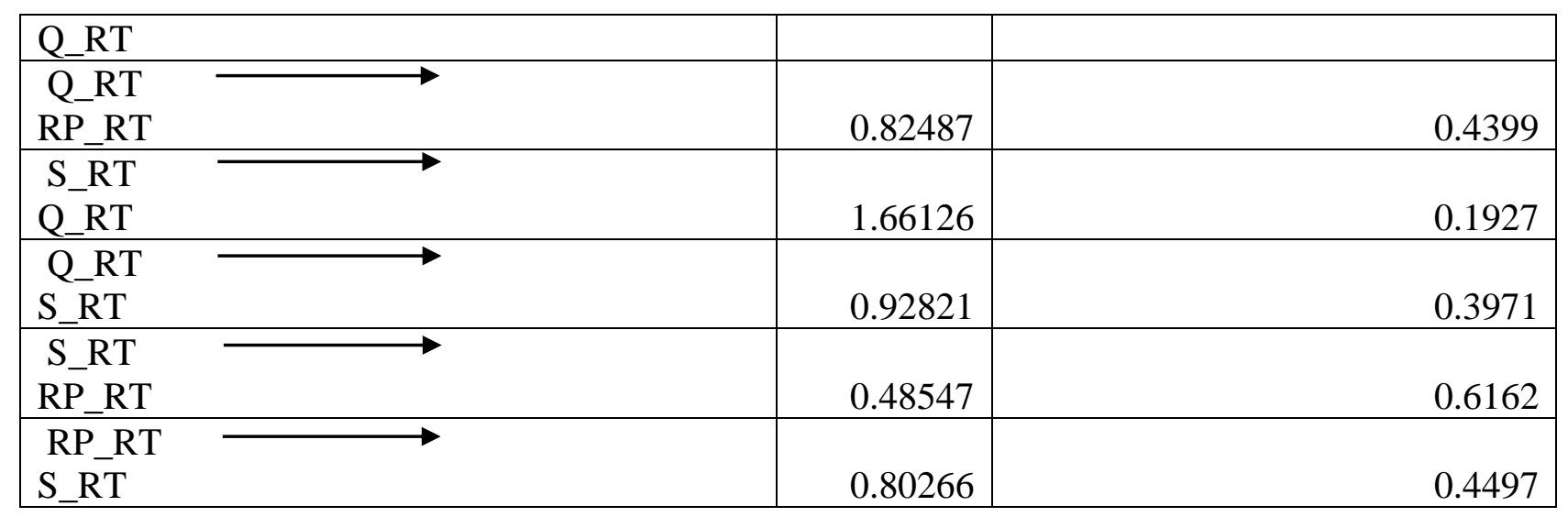

*Significant at level $\mathrm{p}<0.01$

**Significant at level $\mathrm{p}<0.05$

***Significant at level $\mathrm{p}<0.10$

The Change in CPI of China leads to Change in CPI of Pakistan at $p<0.10$. and change in relative price of Pakistan leads to Change in CPI of Pakistan. The change in relative price of China leads to Change in CPI of China. The change in relative price leads to Change in real exchange rate. There exist lead lag relationship in between the PPP variables of Pakistan and China. Table $5 \mathrm{~b}$ indicates that no lead lag and causes of Cpi of Pakistan, real exchange rate, nominal exchange rate and relative prices and Iran same variables of PPP.

Table 5 b. : Pair wise Pakistan versus Iran Causality Test:

\begin{tabular}{|c|c|c|}
\hline Null Hypothesis: & F-Statistic & Probability \\
\hline $\begin{array}{l}\text { CPI_IRN_RT } \\
\text { CPI_PK_RT }\end{array}$ & 0.89667 & 0.4097 \\
\hline $\begin{array}{l}\text { CPI_PK_RT } \\
\text { CPI_IRN_RT }\end{array}$ & 0.31859 & 0.7276 \\
\hline $\begin{array}{l}\text { Q_RT } \longrightarrow \\
\text { CPI_PK_RT }\end{array}$ & 0.03569 & 0.9649 \\
\hline CPI_PK_RT & 1.00749 & 0.3671 \\
\hline $\begin{array}{l}\text { RP_RT -RT } \\
\text { CPI_PK_RT }\end{array}$ & 0.00365 & 0.9964 \\
\hline $\begin{array}{l}\text { CPI_PK_RT } \\
\text { RP_RT }\end{array}$ & 0.16381 & 0.849 \\
\hline $\begin{array}{l}\text { S_RT } \longrightarrow \\
\text { CPI_PK_RT }\end{array}$ & 0.03426 & 0.9663 \\
\hline CPI_PK_RT $\longrightarrow$ & 1.18379 & 0.3084 \\
\hline $\begin{array}{l}\text { Q_RT } \longrightarrow \\
\text { CPI_IRN_RT }\end{array}$ & 0.00128 & 0.9987 \\
\hline CPI IRN RT & 1.34512 & 0.263 \\
\hline $\begin{array}{l}\text { RP_RT } \longrightarrow \\
\text { CPI_IRN_RT }\end{array}$ & 0.00712 & 0.9929 \\
\hline $\begin{array}{l}\text { CPI_IRN_RT } \\
\text { RP_RT }\end{array}$ & 0.22084 & 0.8021 \\
\hline $\begin{array}{l}\text { S_RT } \longrightarrow \\
\text { CPI_IRN_RT }\end{array}$ & 0.00212 & 0.9979 \\
\hline
\end{tabular}




\begin{tabular}{|c|c|c|}
\hline CPI_IRN_RT $\longrightarrow \quad$ S_RT & 1.4264 & 0.2428 \\
\hline $\begin{array}{l}\text { RP_RT } \longrightarrow \\
\text { Q_RT }\end{array}$ & 0.36398 & 0.6954 \\
\hline $\begin{array}{ll}\text { Q_RT } & \longrightarrow \\
\text { RP_RT } & \end{array}$ & 0.47624 & 0.6219 \\
\hline $\begin{array}{l}\text { S_RT } \\
\text { Q_RT } \\
\end{array}$ & 0.60658 & 0.5463 \\
\hline $\begin{array}{ll}\text { Q_RT } & \longrightarrow \\
\text { S_RT }\end{array}$ & 0.67498 & 0.5104 \\
\hline $\begin{array}{ll}\text { S_RT } & \longrightarrow \\
\text { RP_RT } & \end{array}$ & 0.44077 & 0.6442 \\
\hline $\begin{array}{ll}\text { RP_RT } \\
\text { S_RT }\end{array}$ & 0.32104 & 0.7258 \\
\hline
\end{tabular}

at level $\mathrm{p}<0.05$

$* * *$ Significant at level $\mathrm{p}<0.10$

There is no lead lag relationship in between the PPP variables of Pakistan and Turkey. No bidirectional causality exist among the variables.

Table 5 c.: Pair wise Pakistan versus Turkey Causality Test:

\begin{tabular}{|c|c|c|}
\hline Null Hypothesis: & F-Statistic & Probability \\
\hline $\begin{array}{l}\text { CPI_TUR_RT } \\
\text { CPI_PK_RT }\end{array}$ & 8.97507 & 0.000000 \\
\hline $\begin{array}{l}\text { CPI_PK_RT- } \\
\text { CPI_TUR_RT }\end{array}$ & 1.66356 & 0.145800 \\
\hline $\begin{array}{l}\text { Q_RT } \\
\text { CPI_PK_RT }\end{array}$ & 0.06786 & 0.996800 \\
\hline CPI_PK_RT $\longrightarrow$ & 0.61903 & 0.685500 \\
\hline $\begin{array}{l}\text { RP_RT } \\
\text { CPI_PK_RT }\end{array}$ & 3.36619 & $0.006300 *$ \\
\hline $\begin{array}{l}\text { CPI_PK_RT } \longrightarrow \\
\text { RP_RT }\end{array}$ & 3.40201 & $0.005900 *$ \\
\hline $\begin{array}{l}\text { S_RT } \\
\text { CPI_PK_RT }\end{array}$ & 0.09417 & 0.993000 \\
\hline $\begin{array}{l}\text { CPI_PK_RT } \\
\text { S_RT }\end{array}$ & 0.60366 & 0.697200 \\
\hline $\begin{array}{l}\text { Q_RT } \longrightarrow \text { PI_TUR_RT } \\
\text { CPI_TUR }\end{array}$ & 0.08133 & 0.995100 \\
\hline CPI_TUR_RT $\longrightarrow$ & 6.07228 & $0.000030 *$ \\
\hline $\begin{array}{l}\text { RP_RT } \\
\text { CPI_TUR_RT }\end{array}$ & 1.00314 & 0.417400 \\
\hline $\begin{array}{l}\text { CPI_TUR_RT } \\
\text { RP_RT }\end{array}$ & 7.80617 & $0.000001 *$ \\
\hline $\begin{array}{l}\text { S_RT } \longrightarrow \text { PIITUR_RT } \\
\text { CPI_TUR }\end{array}$ & 0.0772 & 0.995600 \\
\hline CPI_TUR_RT $\longrightarrow$ & 5.82341 & $0.000050 *$ \\
\hline $\begin{array}{ll}\text { RP_RT } \\
\text { Q_RT }\end{array}$ & 1.36261 & 0.2406 \\
\hline$\longrightarrow$ & 0.67971 & 0.6394 \\
\hline
\end{tabular}




\begin{tabular}{|cr|r|r|}
\hline RP_RT & & & \\
\hline S_RT & & & \\
Q_RT & & 4.72329 & $0.0004 *$ \\
\hline Q_RT & S_RT & 4.76353 & $0.0004 *$ \\
\hline S_RT & & & \\
RP_RT & & 0.74234 & 0.5927 \\
\hline RP_RT $\longrightarrow$ & S_RT & 1.42578 & 0.2171 \\
\hline
\end{tabular}

*Significant at level $\mathrm{p}<0.01$

$* *$ Significant at level $\mathrm{p}<0.05$

$* * *$ Significant at level $\mathrm{p}<0.10$

The Change in CPI of Turkey leads to Change in CPI of Pakistan at $p<0.10$. and change in relative price of Pakistan leads to Change in CPI of Pakistan. The change in relative price of Turkey leads to Change in CPI of Turkey. The Change in real exchange rate and nominal exchange rate of Pakistan and Turkey. The study indicates that there is long run PPP exist in between Pakistan, China, Iran and Turkey. Table 6 a indicates the results of Vector Error Correction Model between Pakistan, China, Iran and Turkey.

Table 6 a.: Vector Error Correction Model of Pakistan and China:

\begin{tabular}{|c|c|c|c|c|c|}
\hline & D(CPI_PK_RT) & D(CPI_CHI_RT) & D(Q_RT) & D(RP_RT) & $\mathrm{D}\left(\mathrm{S} \_\mathrm{RT}\right)$ \\
\hline \multirow[t]{3}{*}{ CointEq1 } & -0.14567 & -0.01859 & 0.353239 & -110.78 & 0.256983 \\
\hline & -0.07162 & -0.05926 & -0.14083 & -11.5087 & -0.10421 \\
\hline & {$[-2.0340]$} & {$[-0.316]$} & [2.5083] & {$[-9.6257]$} & [2.4660] \\
\hline \multirow[t]{3}{*}{ D(CPI_PK_RT(-1)) } & -0.63913 & 0.061755 & -0.23941 & 39.21027 & -0.33201 \\
\hline & -0.30096 & -0.24901 & -0.5918 & -48.3633 & -0.43791 \\
\hline & {$[-2.12365]$} & {$[0.24800]$} & $\begin{array}{l}{[-} \\
0.40454]\end{array}$ & [ 0.81074] & {$[-0.7581]$} \\
\hline \multirow[t]{3}{*}{ D(CPI_PK_RT(-2)) } & -0.36332 & -0.40269 & 0.698248 & 7.385058 & 0.963113 \\
\hline & -0.29969 & -0.24796 & -0.5893 & -48.1587 & -0.43606 \\
\hline & {$[-1.21234]$} & {$[-1.62402]$} & $\begin{array}{l}\text { [ } \\
1.18488]\end{array}$ & [ 0.15335$]$ & [ 2.2086] \\
\hline \multirow[t]{3}{*}{ D(CPI_CHI_RT(-1)) } & 0.228753 & -0.78202 & -0.22069 & 17.92264 & 0.100893 \\
\hline & -0.28118 & -0.23265 & -0.55291 & -45.1852 & -0.40913 \\
\hline & {$[0.81354]$} & {$[-3.36136]^{*}$} & $\begin{array}{l}{[-} \\
0.39915] \\
\end{array}$ & [0.39665] & [ 0.2466$]$ \\
\hline \multirow[t]{3}{*}{ D(CPI_CHI_RT(-2)) } & 0.142148 & -0.05415 & -1.06489 & 0.821769 & -1.17824 \\
\hline & -0.28533 & -0.23608 & -0.56107 & -45.8523 & -0.41517 \\
\hline & [0.49818] & {$[-0.22937]$} & $\begin{array}{l}{[-} \\
1.89794]\end{array}$ & [0.01792] & $\begin{array}{l}{[-} \\
2.8379]^{*}\end{array}$ \\
\hline \multirow[t]{3}{*}{ D(Q_RT(-1)) } & -0.02117 & 0.074319 & -0.90576 & 40.03413 & -0.38826 \\
\hline & -0.36158 & -0.29917 & -0.71101 & -58.105 & -0.52612 \\
\hline & {$[-0.05855]$} & [ 0.24842] & $\begin{array}{l}{[-} \\
1.27391]\end{array}$ & [ 0.68900$]$ & {$[-0.7379]$} \\
\hline \multirow[t]{3}{*}{ D(Q_RT(-2)) } & 0.090395 & -0.37574 & 0.664722 & 15.01808 & 1.35592 \\
\hline & -0.36349 & -0.30076 & -0.71477 & -58.4127 & -0.5289 \\
\hline & [ 0.24868$]$ & {$[-1.24931]$} & $\begin{array}{l}{[} \\
0.92998]\end{array}$ & [ 0.25710$]$ & $\begin{array}{l}{[} \\
2.5636]^{*}\end{array}$ \\
\hline
\end{tabular}




\begin{tabular}{|c|c|c|c|c|c|}
\hline D(RP_RT(-1)) & 0.000884 & 7.34E-05 & -0.0027 & 0.288353 & -0.00207 \\
\hline & -0.00074 & -0.00062 & -0.00146 & -0.11961 & -0.00108 \\
\hline & [ 1.18808] & [ 0.11923$]$ & $\begin{array}{l}- \\
1.84579]\end{array}$ & $\begin{array}{l}\text { [ } \\
\text { 2.41075]* }\end{array}$ & [-1.9126] \\
\hline \multirow[t]{3}{*}{ D(RP_RT(-2)) } & 0.000309 & 0.000357 & -0.00058 & 0.106494 & -0.00058 \\
\hline & -0.00048 & -0.00039 & -0.00094 & -0.07658 & -0.00069 \\
\hline & [ 0.64851$]$ & [0.90615] & $\begin{array}{l}- \\
0.61349]\end{array}$ & [ 1.39058$]$ & {$[-0.8392]$} \\
\hline \multirow[t]{3}{*}{$\mathrm{D}\left(\mathrm{S} \_\mathrm{RT}(-1)\right)$} & -0.02409 & -0.07953 & 0.335379 & -49.9528 & -0.21512 \\
\hline & -0.36316 & -0.30048 & -0.71411 & -58.3585 & -0.52841 \\
\hline & {$[-0.06633]$} & {$[-0.26468]$} & $\begin{array}{l} \\
0.46965]\end{array}$ & {$[-0.85596]$} & {$[-0.4071]$} \\
\hline \multirow{3}{*}{$\mathrm{D}\left(\mathrm{S} \_\mathrm{RT}(-2)\right)$} & -0.0953 & 0.371511 & -1.04935 & -24.2704 & -1.76323 \\
\hline & -0.36491 & -0.30193 & -0.71756 & -58.6403 & -0.53096 \\
\hline & {$[-0.26115]$} & [ 1.23047$]$ & $\begin{array}{l}{[-} \\
1.46240]\end{array}$ & {$[-0.41389]$} & $\begin{array}{l}{[-} \\
\text { 3.3208] }\end{array}$ \\
\hline \multirow[t]{3}{*}{$\mathrm{C}$} & $8.54 \mathrm{E}-06$ & $-7.80 \mathrm{E}-06$ & $8.40 \mathrm{E}-05$ & 0.001047 & 0.000104 \\
\hline & -0.00012 & -0.0001 & -0.00024 & -0.01982 & -0.00018 \\
\hline & [ 0.06927$]$ & {$[-0.07646]$} & $\begin{array}{l}{[} \\
0.34626]\end{array}$ & [ 0.05284$]$ & [ 0.5788$]$ \\
\hline R-Squared & 0.400673 & 0.424226 & 0.397003 & 0.609087 & 0.421028 \\
\hline Adj. R-Squared & 0.363216 & 0.38824 & 0.359315 & 0.584654 & 0.384843 \\
\hline F- Statistic & 10.69663 & 11.78866 & 10.53412 & 24.92977 & 11.6352 \\
\hline
\end{tabular}

*significant at [t>1.96]

Vector error correction model is being used for evaluating the short term relationship among PPP variables. Whereas t-statistics at $t>1.96$ indicates the presence of short run relationship between the relevant variables and there held short run relationship within economies of Pakistan and China as Real exchange rate and CPI is indicating.

Table 6 b.: Vector Error Correction Model of Pakistan and Iran:

\begin{tabular}{|c|c|c|c|c|c|}
\hline & D(CPI_PK_RT) & D(CPI_CHI_RT) & D(Q_RT) & D(RP_RT) & $\mathrm{D}\left(\mathrm{S} \_\mathrm{RT}\right)$ \\
\hline \multirow[t]{3}{*}{ CointEq1 } & -0.59441 & -0.68333 & 4.866503 & 3.20503 & 4.849232 \\
\hline & -1.59179 & -1.59028 & -1.90842 & -7.03955 & -1.95708 \\
\hline & {$[-0.37342]$} & {$[-0.42969]$} & $\begin{array}{l}\text { [ } \\
2.55002]\end{array}$ & [ 0.45529$]$ & $\begin{array}{l} \\
2.47779]\end{array}$ \\
\hline \multirow[t]{3}{*}{ D(CPI_PK_RT(-1)) } & 4.324628 & 5.043004 & -2.13119 & -3.08845 & -2.40969 \\
\hline & -3.78799 & -3.7844 & -4.54147 & -16.7521 & -4.65727 \\
\hline & [ 1.14167] & [ 1.33258$]$ & $\begin{array}{l}{[-} \\
0.46927]\end{array}$ & {$[-0.18436]$} & $\begin{array}{l}{[-} \\
0.51740]\end{array}$ \\
\hline \multirow[t]{3}{*}{ D(CPI_PK_RT(-2)) } & 8.667555 & 9.298182 & 6.69429 & 13.38171 & 7.005369 \\
\hline & -3.62475 & -3.62131 & -4.34576 & -16.0301 & -4.45656 \\
\hline & {$[2.39122] *$} & {$[2.56763]^{*}$} & $\begin{array}{l}\text { [ } \\
1.54042]\end{array}$ & {$[0.83478]$} & $\begin{array}{l}{[} \\
1.57192]\end{array}$ \\
\hline \multirow[t]{3}{*}{ D(CPI_IR_RT(-1)) } & 2.590164 & 2.138267 & 7.783831 & 4.142884 & 7.991236 \\
\hline & -3.29298 & -3.28986 & -3.948 & -14.5629 & -4.04866 \\
\hline & [ 0.78657$]$ & [ 0.64996$]$ & $\begin{array}{l}\text { [ } \\
1.97159]\end{array}$ & [ 0.28448$]$ & $\begin{array}{l}\text { [ } \\
\text { 1.97380]* }\end{array}$ \\
\hline
\end{tabular}




\begin{tabular}{|c|c|c|c|c|c|}
\hline D(CPI_IR_RT(-2)) & -0.30568 & -0.53739 & 3.38453 & -10.6925 & 3.495645 \\
\hline & -3.13367 & -3.1307 & -3.757 & -13.8584 & -3.85279 \\
\hline & {$[-0.09755]$} & {$[-0.17165]$} & $\begin{array}{l} \\
0.90086]\end{array}$ & {$[-0.77155]$} & $\begin{array}{l}\text { [ } \\
0.90730]\end{array}$ \\
\hline \multirow[t]{3}{*}{ D(Q_RT(-1)) } & -1.71376 & -1.73123 & 2.454117 & 3.714891 & 3.166173 \\
\hline & -2.28314 & -2.28098 & -2.73729 & -10.097 & -2.80708 \\
\hline & {$[-0.75062]$} & {$[-0.75899]$} & $\begin{array}{l} \\
0.89655]\end{array}$ & [ 0.36792$]$ & $\begin{array}{l}\text { [ } \\
1.12792]\end{array}$ \\
\hline \multirow[t]{3}{*}{ D(Q_RT(-2)) } & -2.68308 & -2.66033 & -0.33065 & -12.1111 & 0.085858 \\
\hline & -1.89767 & -1.89587 & -2.27515 & -8.3923 & -2.33316 \\
\hline & {$[-1.41388]$} & {$[-1.40322]$} & $\begin{array}{l}{[-} \\
0.14533] \\
\end{array}$ & {$[-1.44312]$} & $\begin{array}{l} \\
0.03680] \\
\end{array}$ \\
\hline \multirow[t]{3}{*}{ D(RP_RT(-1)) } & -0.00336 & -0.00407 & 0.052164 & -0.83523 & 0.05214 \\
\hline & -0.02214 & -0.02212 & -0.02655 & -0.09793 & -0.02723 \\
\hline & {$[-0.15189]$} & {$[-0.18398]$} & [1.96478]* & $\begin{array}{l}{[-} \\
8.52847]^{*}\end{array}$ & [ \\
\hline \multirow[t]{3}{*}{ D(RP_RT(-2)) } & -0.00622 & -0.00649 & 0.025898 & -0.29746 & 0.025812 \\
\hline & -0.01797 & -0.01795 & -0.02154 & -0.07946 & -0.02209 \\
\hline & {$[-0.34639]$} & {$[-0.36176]$} & $\begin{array}{l}{[} \\
1.20220]\end{array}$ & $\begin{array}{l}{[-} \\
3.74340]^{*}\end{array}$ & $\begin{array}{l}\text { [ } \\
1.16842]\end{array}$ \\
\hline \multirow[t]{3}{*}{$\mathrm{D}\left(\mathrm{S} \_\mathrm{RT}(-1)\right)$} & 1.571973 & 1.581688 & -2.72682 & -3.22824 & -3.44009 \\
\hline & -2.07951 & -2.07754 & -2.49316 & -9.19647 & -2.55673 \\
\hline & [ 0.75593$]$ & [ 0.76133] & $\begin{array}{l}{[-} \\
1.09372]\end{array}$ & {$[-0.35103]$} & $\begin{array}{l}{[-} \\
1.34551]\end{array}$ \\
\hline \multirow[t]{3}{*}{$\mathrm{D}\left(\mathrm{S} \_\mathrm{RT}(-2)\right)$} & 2.435447 & 2.413763 & 0.010217 & 10.80529 & -0.40426 \\
\hline & -1.75084 & -1.74918 & -2.09911 & -7.74296 & -2.15263 \\
\hline & [ 1.39101] & [ 1.37994] & $\begin{array}{l}\text { [ } \\
0.00487]\end{array}$ & [ 1.39550$]$ & $\begin{array}{l}{[-} \\
0.18780]\end{array}$ \\
\hline \multirow[t]{3}{*}{$\mathrm{C}$} & -0.00519 & -0.00522 & -0.0049 & -0.00498 & -0.00489 \\
\hline & -0.00534 & -0.00533 & -0.0064 & -0.02361 & -0.00656 \\
\hline & {$[-0.97112]$} & {$[-0.97907]$} & $\begin{array}{l}{[-} \\
0.76560]\end{array}$ & {$[-0.21072]$} & $\begin{array}{l}- \\
0.74472]\end{array}$ \\
\hline R-Squared & 0.042558 & 0.044675 & 0.232318 & 0.515578 & 0.243448 \\
\hline Adj. R-Squared & -0.01694 & -0.0147 & 0.184609 & 0.485472 & 0.19643 \\
\hline F- Statistic & 0.715239 & 0.752477 & 4.869488 & 17.12578 & 5.177821 \\
\hline
\end{tabular}

*significant at [t>1.96]

Vector error correction model is being used for evaluating the short term relationship among PPP variables. Whereas t-statistics at $t>1.96$ indicates the presence of short run relationship between the relevant variables and there held short run relationship within economies of Pakistan and Iran.

Table 6 c.: Vector Error Correction Model of Pakistan and Turkey:

\begin{tabular}{||l|r|r|r|r|r||}
\hline \multicolumn{7}{||c||}{} \\
\hline & D(CPI_PK_RT) & D(CPI_CHI_RT) & D(Q_RT) & D(RP_RT) & D(S_RT) \\
\hline CointEq1 & 4.648885 & 4.920248 & 26.89385 & 8.617406 & 29.35863 \\
\hline & -7.77434 & -7.77063 & -10.2215 & -8.92739 & -10.565 \\
\hline & {$[0.59798]$} & {$[0.63319]$} & {$[2.63111]$} & {$[0.96528]$} & $2.77886]$ \\
\hline
\end{tabular}




\begin{tabular}{|c|c|c|c|c|c|}
\hline \multirow{2}{*}{ D(CPI_PK_RT(-1)) } & -0.558386 & -0.15605 & -22.08882 & 0.169659 & -24.04587 \\
\hline & -8.67528 & -8.67114 & -11.406 & -9.96195 & -11.7893 \\
\hline & {$[-0.06437]$} & {$[-0.01800]$} & {$[-1.93659]$} & [ 0.01703$]$ & $2.03963]^{[-}$ \\
\hline \multirow[t]{3}{*}{ D(CPI_PK_RT(-2)) } & 5.841176 & 5.998417 & -5.243736 & 2.769047 & -6.095168 \\
\hline & -7.57912 & -7.5755 & -9.96483 & -8.70321 & -10.2997 \\
\hline & [ 0.77069] & [ 0.79182] & {$[-0.52622]$} & [ 0.31816$]$ & $\begin{array}{r}{[-} \\
0.59178]\end{array}$ \\
\hline \multirow[t]{3}{*}{ D(CPI_TUR_RT(-1)) } & -0.344596 & -0.38139 & 20.26698 & 5.480655 & 21.60715 \\
\hline & -8.92122 & -8.91695 & -11.7294 & -10.2444 & -12.1236 \\
\hline & {$[-0.03863]$} & {$[-0.04277]$} & [ 1.72788$]$ & [ 0.53499$]$ & $1.78224]$ \\
\hline \multirow[t]{3}{*}{ D(CPI_TUR_RT(-2)) } & 5.536287 & 5.582842 & 16.22742 & 12.68664 & 16.73 \\
\hline & -7.74003 & -7.73634 & -10.1764 & -8.88799 & -10.5184 \\
\hline & [ 0.71528$]$ & [ 0.72164$]$ & [ 1.59461] & [ 1.42739] & $1.59055]$ \\
\hline \multirow[t]{3}{*}{$\mathrm{D}\left(\mathrm{Q} \_\mathrm{RT}(-1)\right)$} & -0.617438 & -0.827274 & -16.18042 & -3.502068 & -16.88502 \\
\hline & -5.00706 & -5.00467 & -6.58315 & -5.74968 & -6.80438 \\
\hline & {$[-0.12331]$} & {$[-0.16530]$} & $\begin{array}{r}{[-} \\
2.45785]^{*}\end{array}$ & {$[-0.60909]$} & $\begin{array}{r}{[-} \\
2.48149]^{*}\end{array}$ \\
\hline \multirow[t]{3}{*}{$\mathrm{D}\left(\mathrm{Q} \_\mathrm{RT}(-2)\right)$} & 9.498625 & 9.188814 & 1.037679 & 3.998714 & 1.075948 \\
\hline & -3.75554 & -3.75375 & -4.93769 & -4.31254 & -5.10362 \\
\hline & [2.52923]* & [2.44790]* & [ 0.21015$]$ & [ 0.92723$]$ & $0.21082]$ \\
\hline \multirow[t]{3}{*}{ D(RP_RT(-1)) } & -0.036937 & -0.032934 & -0.146251 & -0.395962 & -0.157971 \\
\hline & -0.35812 & -0.35795 & -0.47084 & -0.41123 & -0.48667 \\
\hline & {$[-0.10314]$} & {$[-0.09201]$} & {$[-0.31062]$} & {$[-0.96287]$} & $\begin{array}{r}{[-} \\
0.32460] \\
\end{array}$ \\
\hline \multirow[t]{3}{*}{ D(RP_RT(-2)) } & -0.583516 & -0.574788 & -0.544987 & -0.778255 & -0.557317 \\
\hline & -0.35175 & -0.35158 & -0.46247 & -0.40392 & -0.47801 \\
\hline & {$[-1.65890]$} & {$[-1.63486]$} & {$[-1.17842]$} & [-1.92676] & $\begin{array}{r}{[-} \\
1.16591]\end{array}$ \\
\hline \multirow[t]{3}{*}{$\mathrm{D}\left(\mathrm{S} \_\mathrm{RT}(-1)\right)$} & 0.611607 & 0.810506 & 14.34725 & 3.349279 & 14.94912 \\
\hline & -4.6716 & -4.66937 & -6.1421 & -5.36447 & -6.34851 \\
\hline & [ 0.13092$]$ & {$[0.17358]$} & [2.33589] & {$[0.62434]$} & $2.35474]^{[}$ \\
\hline \multirow[t]{3}{*}{ D(S_RT(-2)) } & -8.77438 & -8.489258 & -1.345183 & -3.743839 & -1.404413 \\
\hline & -3.48754 & -3.48588 & -4.58533 & -4.0048 & -4.73943 \\
\hline & {$[-2.51592]^{*}$} & {$[-2.43533]^{*}$} & {$[-0.29337]$} & {$[-0.93484]$} & $\begin{array}{r}\text { [- } \\
0.29633] \\
\end{array}$ \\
\hline \multirow[t]{3}{*}{$\mathrm{C}$} & -0.004868 & -0.004895 & -0.005475 & -0.005157 & -0.005498 \\
\hline & -0.00505 & -0.00505 & -0.00664 & -0.0058 & -0.00686 \\
\hline & {$[-0.96377]$} & {$[-0.96940]$} & {$[-0.82431]$} & {$[-0.88896]$} & $\begin{array}{r}{[-} \\
0.80092]\end{array}$ \\
\hline R-Squared & 0.143617 & 0.14299 & 0.39745 & 0.182957 & 0.413257 \\
\hline Adj. R-Squared & 0.090395 & 0.089729 & 0.360003 & 0.13218 & 0.376793 \\
\hline F- Statistic & 2.69847 & 2.684725 & 10.61376 & 3.603172 & 11.33323 \\
\hline
\end{tabular}

*significant at [t>1.96] 
Vector error correction model is being used for evaluating the short term relationship among PPP variables. Whereas t-statistics at $\mathrm{t}>1.96$ indicates the presence of short run relationship between the relevant variables and there held short run relationship within economies of Pakistan and Turkey.

\section{Conclusion}

This study is aimed at to test the Validity of PPP theory and dynamics of exchange rate behavior between Pakistan and the economies of China, Iran and Turkey. The deviations from PPP are due to structural changes in real exchange rate. The PPP does not hold due to difference at transaction cost i.e. prices of goods, carrying cost, administration, custom taxes and tariffs. The difference at transaction cost level is being violated the parity conditions and law of one price does not hold under PPP. The transaction cost and law of one price is also being producer of growth and efficiency of the economy. The difference in growth and efficiency of countries Pakistan, China, Iran and Turkey is very important to consider and to develop policies accordingly. However results reveal that there exists long run relationship exists among the exchange rate dynamics of Pakistan with China, Iran and Turkey. Results are in alignment with the study of Froot and Rogoff (1995), Bhatti (1996) Arize et. al, (2004) but not in accordance with the study of Alba and Park (2005). However, there exist little evidences about short run relationship. The decisions of future can be made through the behaviour exchange rate. PPP is very helpful in an estimation of exchange rate behavior as identified by $\mathrm{Li}$ and Park (2017). The increase in inflation can cause to depreciate the home currency. The development of monetary policy can have implications to preserve the value of currency. The value of national currency and foreign trade strategies are actually based on PPP. The PPP must hold at long term due to tendency of currencies towards faire value sustained at long term. The faire value can be created through an arbitraging process "buying currency at low value and selling currency at high value. This under value and over value process must be profitable with better managing of exchange rate risk and ultimately settled at fair value. The optimization of currency level with reference to political and economic stability is also considerable factor for valid PPP in future.

\section{Reference}

Alba. D. J, and Park. D., (2005). An empirical investigation of purchasing power parity (PPP) for Turkey, Journal of Policy Modeling, 989-1000.

Arize. C. A, Malindretos. J and Grivoyannis. C. E, (2004). Purchasing power parity in developing countries: Evidence from conventional and fractional co-integration tests, 2 (1). 29-43.

Bhatti. R. H, (1996). A correct test of purchasing power parity. The Case of Pak-Rupee exchange rate", The Pakistan Development Review , 671-682.

Cheung, Yin-Wong and Lai, Kon S. (1994). Mean Reversion in Real Exchange Rates, Economic Letters, 46(3), pp.251-56.

Chortareas, G., \& Kapetanios, G. (2013). How Puzzling Is The PPP Puzzle? An Alternative Half-Life Measure of Convergence to PPP. Journal of Applied Econometrics, 28(3), 435-457.

Dickey, D. A, and W. A. Fuller, (1981), "Likelihood Ratio Statistics for Autoregressive time series with Unit root", Econometrica, 49, pp.1057-1072

Dixon, R. Zhang, Z. and Dai, Y., (2016). Exchange rate flexibility in China: measurement, regime shifts and driving forces of change. Review of International Economics, 24, pp. 875-892.

Elsadig, A. (2011). Testing the evidence of purchasing power parity for Asian-5 countries using panel estimation. International Journal of Economics and Business Modeing. 2(1), 42-56.

Engel, R. F., and C. W. J. Granger, (1987). Co-integration error correction representation estimation and testing, Econometrica, 55:1251-1276

Froot, K. A., \& Rogoff, K. (1995). Perspectives on PPP and long-run real exchange rates (No. w4952). National Bureau of Economic Research.

Glenville, R. (2013). Inflation and purchasing power parity in South Africa. The journal of Applied Business and Economics. 15(3), 11-18.

He, B.L. Zhu, H.X. Chen, D.B. and Shi, Y., 2015. On Pass-through of RMB Exchange Rate to Prices

of Different Industries. Procedia Computer Science, 55, pp. 886-895.

Johansen, S. (1988). Statistical analysis of cointegration vectors. Journal of Economic Dynamics and Control, 12(2), 231-254. 
Johansen, S., \&Juselius, K. (1990). Maximum likelihood estimation and inference on cointegration with applications to the demand for money. Oxford Bulletin of Economics and Statistics, 52(2), 169-210.

Kanyembo, C. and Sheefeni, J.P. (2013). Purchasing Power Parity between Zambia and South

Africa, Journal of Emerging Issues in Economics, Finance and Banking (JEIEFB), 2, 858-

871.

Khan, M. A., \& Qayyum, A. (2007). Exchange rate determination in Pakistan: Evidence based on purchasing power parity theory. Pakistan Economic and Social Review, 181-202.

Ma, W. Li, H.Q. and Park, S.Y., (2017). Empirical conditional quantile test for purchasing power parity: Evidence from East Asian countries. International Review of Economics and

Finance, 49:211-222.

Murray. J. C and Papell. H. D, (2002). The purchasing power parity persistence paradigm, Journal of

International Economics (1-19).

Phillips, P., and P. Perron, (1988). Testing for a unit root in time series regression, Biometrica, 75:335-346

Sercu, P., Uppal, R., \& Van Hulle, C. (1995). The exchange rate in the presence of transaction costs: implications for tests of purchasing power parity. Journal of Finance, 1309-1319.

Sarno, L. and Taylor, M.P. (2002). The Economics of Exchange Rates, Cambridge University Press, Cambridge.

Shively, P.A, (2001). A Test of Long-Run Purchasing Power Parity”, Economics Letters, 73,201205.

Taylor. M. A, (2002). A Century of Purchasing Power Parity” The Review of Economics and

Statistics, February 2002, 84(1): 139-150

Taylor, M.P. \& Sarno, L. (1998). The behavior of real exchange rates during the post-Bretton Woods period, Journal of International Economics, (1998), Vol. 46, pp. 281-312.

Zyoud, H.A. (2015). An Empirical Test of Purchasing Power Parity Theory for Canadian Dollar-US

Dollar Exchange Rates, International Journal of Economics and Finance, 7:3, 233-240. 